\title{
Extracorporeal Life Support for Massive Hemoptysis and Acute Lung Injury Due to ANCA- Negative Vasculitis
}

\author{
Joseph A. McGuire ${ }^{1}$, Paul McCarthy ${ }^{2}$, Robert Herron ${ }^{3}$, J.W. Awori Hayanga ${ }^{3}$, Veena \\ Nandwani ${ }^{3}$, James Fugett ${ }^{4}$, Heather K. Hayanga ${ }^{5}$
}

\section{Abstract}

Massive hemoptysis is life-threatening and extracorporeal membrane oxygenation (ECMO) may be indicated as rescue therapy due to acute lung injury in this setting. We report a case of a patient who presented with massive hemoptysis and acute lung injury after reported inhaled ground oxymorphone abuse. Although initially thought to have been due to inhaled oxymorphone, we determined that the patient had ANCA-negative vasculitis that led to both hemoptysis and hematuria. The role of inhaled oxymorphone in combination with the vasculitis is unknown. ECMO was initiated to support the patient and, although his course was complicated, he did recover.

Keywords: ECMO; Vasculitis; Acute Respiratory Distress (ARDS).

\section{Introduction}

Approximately $10 \%$ of patients presenting with hemoptysis will have massive hemoptysis, defined as pulmonary bleeding of $100 \mathrm{ml}-600 \mathrm{ml}$ within 24 hours [1]. The etiology of massive hemoptysis varies, with drugs and toxins, infections, malignancy, and vasculitis all having been previously reported to cause the condition [2]. Extracorporeal membrane oxygenation (ECMO) can be used to treat patients with acute severe cardiac and pulmonary failure and has been successfully used to support patients with hemoptysis with acute lung injury [3-5]. The international registry maintained by the Extracorporeal Life Support Organization reports a 58\% survival to hospital discharge rate in respiratory failure patients on ECMO [6], demonstrating its efficacy in the most acutely ill.

Vasculitis is a well-known cause of alveolar hemorrhage and hematuria. However, various infections and poisonings may cause a similar presentation [1]. We present a case of a patient who suffered from hemoptysis, hematuria, acute respiratory distress syndrome (ARDS), and acute kidney injury ultimately found to have ANCA- negative vasculitis. The patient was supported with ECMO. His initial diagnosis was confounded by the recent inhalation of ground extended release oxymorphone which may or may not have contributed to the patient's clinical presentation.

\section{Case Report}

A 29-year-old male with a history of Hepatitis C and past intravenous drug use presented to our institution from an outlying facility with profound dyspnea refractory to supplemental oxygen, inhaled nebulizer therapy, and oral steroids. On initial presentation to the outlying facility, he complained of worsening bilateral lower extremity edema, a rash, hemoptysis, hematuria and bilateral knee joint pain. He denied recent intravenous drug use but admitted to inhaling ground extended release oxymorphone. A chest radiograph revealed diffuse patchy airspace consolidation bilaterally (Figure 1). Computed tomography (CT) of the chest showed diffuse patchy airway disease, negative for pulmonary embolus. Laboratory examination showed respiratory acidosis on arterial blood gas (ABG). Due to his reported history of inhaled opioid abuse, the referring facility strongly felt the hemoptysis was the result of his inhaled drug abuse. The patient became increasingly dyspneic and

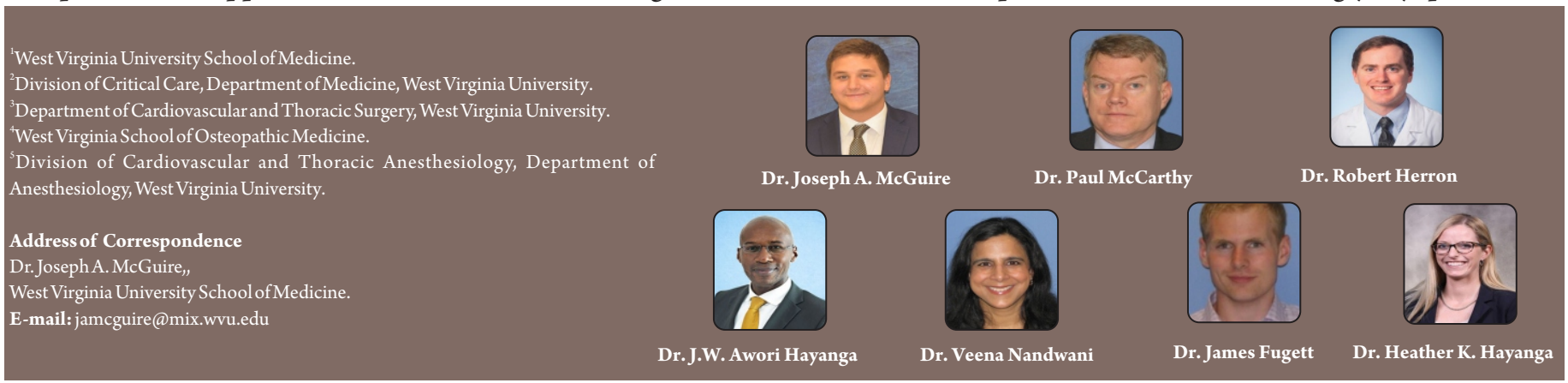

2021 (C) Journal of Anaesthesia and Critical Care Case Reports| Available on www.jaccr.com |DOI: 10.13107/jaccr.2021.v07i01.165

This is an Open Access article distributed under the terms of the Creative Commons Attribution Non-Commercial License (http://creativecommons.org/licenses/by-nc/3.0)

which permits unrestricted non-commercial use, distribution, and reproduction in any medium, provided the original work is properly cited. 


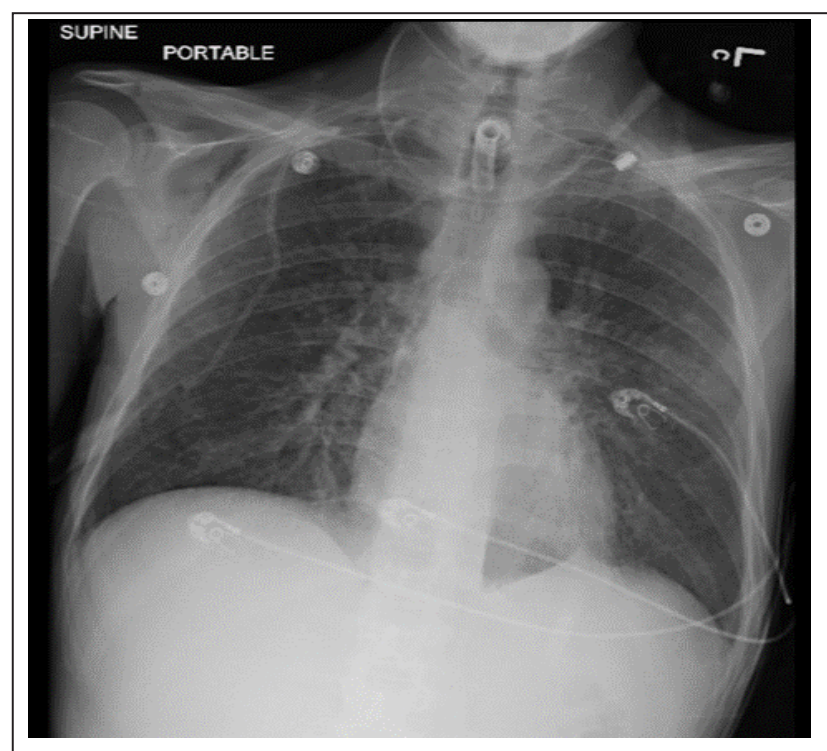

Figure 1: Antero-posterior chest radiograph on day of VV-ECMO cannulation

tachypneic with rapidly worsening respiratory distress and was subsequently intubated immediately prior to transfer. Upon arrival to our intensive care unit, he was profoundly hypoxemic while on a high level of mechanical ventilatory support with evidence of progressing acute respiratory distress syndrome (ARDS). Ventilation settings upon presentation included a fraction of inspired oxygen of $100 \%$ and 18 centimeters of water positive end-expiratory pressure. Initial partial arterial pressure of oxygen $\left(\mathrm{pO}_{2}\right)$ via $\mathrm{ABG}$ was 75 millimeters of mercury $(\mathrm{mmHg}) . \mathrm{PaO}_{2} /$ fraction of inspired oxygen $\left(\mathrm{FiO}_{2}\right)$ ratio was 75 . His chest radiograph upon arrival revealed marked bilateral consolidation and his Murray Score was 3.7, indicating severe acute lung injury. The initial $A B G$ values included: $\mathrm{pH}$ of 7.29 , partial arterial pressure of $\mathrm{CO}_{2}\left(\mathrm{pCO}_{2}\right)$ of $48 \mathrm{mmHg}$, bicarbonate of 22 millimoles/liter $(\mathrm{mmol} / \mathrm{L})$, and a base deficit of 3.8 milliequivalents/liter (mEq/L). Follow-up ABG one hour later revealed a worsening hypoxemic process and respiratory acidosis: $\mathrm{pH} 7.23, \mathrm{pO}_{2} 43 \mathrm{mmHg}, \mathrm{pCO}_{2} 57$ $\mathrm{mmHg}$, bicarbonate $22 \mathrm{mmol} / \mathrm{L}$, base deficit $3.4 \mathrm{mEq} / \mathrm{L}$, and $\mathrm{PaO}_{2} / \mathrm{FiO}_{2}$ ratio of 43 . Oxyhemoglobin saturation was $72.9 \%$ and hemoglobin concentration was $6.1 \mathrm{~g} / \mathrm{dL}$. The patient also was beginning to show some signs of hemodynamic instability. An infusion of norepinephrine was started to keep the patient normotensive. Flexible bronchoscopy revealed copious thick, bloody secretions in all lobes bilaterally which required multiple attempts at aspiration. Considering the bronchoscopic and CT findings, it became apparent the patient was suffering from diffuse, bilateral alveolar hemorrhage. The patient continued to have ongoing intra-alveolar bleeding, requiring transfusion. The diffuse and persistent hemorrhage precluded his ability to adequately oxygenate despite maximal mechanical ventilatory support and conventional ARDS management measures including deep sedation and chemical paralysis. He continued to decompensate rapidly and became increasingly difficult to oxygenate.

Considering the combination of hemoptysis and hematuria, a vasculitis etiology related to pulmonary-renal syndrome was suspected. Due to this, steroids and plasma exchange were implemented. To (plasma exchange was started after ECMO, would place this later in write-up) address his refractory hypoxemia secondary to his diffuse pulmonary injury, the decision was made to institute venovenous ECMO (VV-ECMO) as a bridge to recovery. He was peripherally cannulated emergently in the intensive care unit via right internal jugular and right femoral veins. Soon after initiation of the VV-ECMO circuit, his oxygen saturation rose to $100 \%$ and his hemodynamics improved. He was discovered to have a right pneumothorax and subcutaneous emphysema for which a right chest pigtail catheter was placed.

Following cannulation, the patient required low-dose vasopressors to maintain adequate mean arterial pressure. $\mathrm{He}$ underwent serial flexible bronchoscopies to maintain optimal pulmonary hygiene in view of ongoing diffuse alveolar hemorrhage. Bronchoscopy revealed inflamed airway mucosa involving proximal bronchi and distal tertiary bronchi. An infectious work-up, including bronchoalveolar lavage sampling, urine and blood cultures, did not reveal any infectious etiology. Hematuria was noted and he was found to have 3.4 grams of urinary protein and dysmorphic red blood cells (RBCs). Autoimmune evaluation, including cryoglobulins, yielded no positive results. Due to suspected pulmonary-renal syndrome, in addition to steroids which were started prior to ECMO cannulation, plasma exchange was initiated.

After 3 days on ECMO, his sedation was weaned, revealing a normal neurologic exam. By day 5, his improvement was substantial enough to enable the weaning of ECMO sweep gas. On day 6, he was found to have acute anemia requiring transfusion, continuing hematuria, and non-oliguric acute kidney injury. Plasma exchange was performed again, and the patient was started on continuous renal replacement therapy (CRRT). The copious bloody airway secretions persisted and required serial bronchoscopies for maintenance of pulmonary hygiene. During days 12 to 16 , he showed evidence of disseminated intravascular coagulation (DIC) and thrombosed several CRRT circuits and the ECMO circuit also developed a significant amount of fibrin clots. His DIC seemed to improve after ECMO circuit change that occurred during this time frame. He underwent plasma exchange again thereafter. On day 16 , he was placed on a bivalirudin drip to mitigate the risk of thrombosis within the 

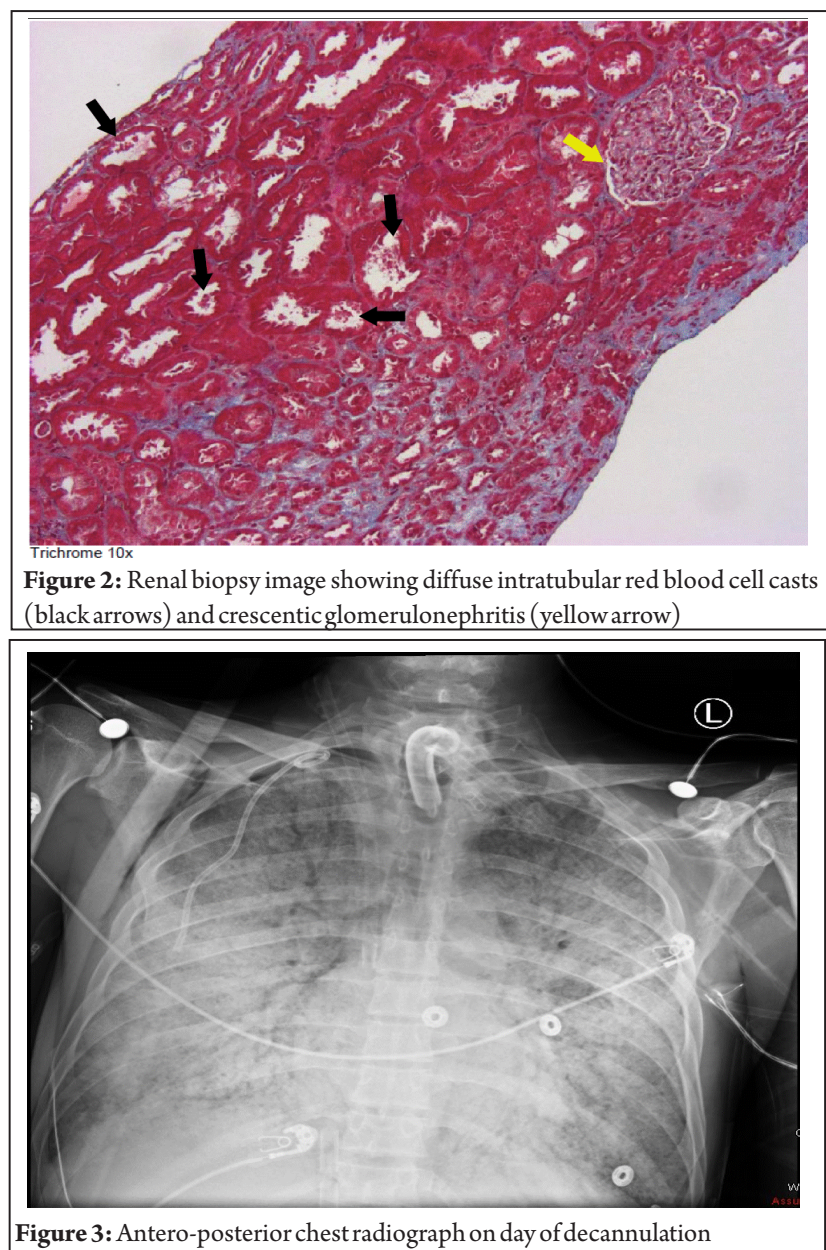

ECMO circuit. On day 19, a renal biopsy, initially delayed due to clinical instability and coagulopathy, demonstrated focal necrotizing and crescentic glomerulonephritis (GN), pauci-immune type (ANCA- negative), and acute tubular injury with intratubular red blood cell casts (Figure 2).

With continued aggressive supportive care, he began to make steady clinical progress. His VV-ECMO support was subsequently weaned to minimal settings and he was successfully decannulated on day 32 (Figure 3). He was transitioned to intermittent hemodialysis from CRRT following decannulation. The patient progressed well throughout the remainder of his hospital course and was transferred to a step-down unit on day 38 . He was discharged to home on day 53 free of the need for hemodialysis.

\section{Discussion}

This is a case demonstrating the utilization of VV-ECMO as a component of comprehensive critical care support (i.e. plasma exchange, lung protective ventilation, nutritional support), in the successful management of a young male who presented with a pulmonary-renal syndrome caused by biopsy-proven ANCA- negative vasculitis. This patient's hemoptysis was initially presented to be due to inhaled ground extended release oxymorphone, however, the differential diagnosis was broadened following discovery of additional symptoms. Case reports exist supporting the use of ECMO for lung injury caused by opioid abuse [7]. However, hemoptysis related to opioids is known to exist in the context of Thrombotic Microangiopathy (TMA) due to rapid hemolysis $[8,9]$.

The presenting picture of this patient posed a challenging scenario for physicians prior to obtaining a kidney biopsy. The presentation suggests pulmonary-renal syndrome secondary to vasculitis or a potential toxic insult from the inhaled oxymorphone, leading to TMA. Both of these pathologies can appear clinically identical; however, the pathology and treatment are quite distinct. The renal biopsy showed no evidence of TMA as has been previously described with intravenous or inhaled oxymorphone. Rather, the biopsy showed the presence of focal necrotizing and crescentic glomerulonephritis (GN), pauci-immune type, confirming a pulmonary-renal syndrome etiology. A question remains unanswered. Did the inhaled oxymorphone contribute to this patient's acute presentation; TMA may have potentially been missed due to sampling error or inhalation of the toxic substance itself may have exacerbated acute lung injury without also causing TMA. Toxins, such as smoke inhalation, have also been implicated in the clinical unmasking of vasculitis probably due to their ability to induce vascular damage $[10,11]$.

Conventional mechanical ventilation techniques were unsuccessful in this patient due to the massive hemoptysis blocking the ability to safely oxygenate and ventilate. It is in scenarios like this that ECMO can be used to enable safer ventilation and oxygenation. ECMO allows a marked reduction in ventilator settings, decreasing the pulmonary damage caused by high airway pressures, whilst allowing for more time to correct underlying pathology [12]. Bleeding and DIC are not uncommon complications with patients on extracorporeal support [13]. This was seen in this patient likely secondary to a consumption of clotting factors. This did improve with a change of the ECMO circuit.

\section{Conclusion}

Comprehensive, meticulous critical care which includes VVECMO may serve as an effective means of treatment as a bridge to recovery in patients who endure severe lung injury due to vasculitis potentially compounded by he inhalation of (a) synthetic compound(s). This modality may prove even more effective and prevalent as the rates of world-wide vasculitis cases continues to rise [2,14]. Astute clinical acumen must entertain a multitude of potential diagnoses when determining etiology to ensure appropriate management to increase the likelihood of successful recovery. 


\section{References}

1. Sakr, L., \& Dutau, H. (2010). Massive hemoptysis: An update on the role of bronchoscopy in diagnosis and management. Respiration; International Review of Thoracic Diseases,

80(1),38-58.https://doi.org/10.1159/000274492

2. Savage, C. O. S., Harper, L., Cockwell, P., Adu, D., \& Howie, A. J. (2000). Vasculitis. BMJ :British Medical Journal,320(7245), 1325-1328.

3. Endicott-Yazdani, T. R., Wood, C., Trinh, A. D., \& Mora, A. (2018). Massive hemoptysis managed by rescue extracorporeal membrane oxygenation. Proceedings (Baylor University. Medical Center), 31(4), 479-481. https://doi.org/10.1080/08998280.2018.1487693

4. Yoo, J.-H., Kang, H. M., Choi, C. W., Park, M. J., \& Lee, J.H.

(2012). Extracorporeal membrane oxygenation as bridge in patients with non-iatrogenic massive hemoptysis. European Respiratory Journal,

40(Suppl 56).https://erj.ersjournals.com/content/40/Suppl_56/P1996

5. Kazuma, S. (2016). Almost Total Airway Obstruction Due to Unidentified Massive Hemoptysis under Extracorporeal Membrane Oxygenation. International Journal of Critical Care and Emergency Medicine, 2(1). https://doi.org/10.23937/2474-3674/1510012

6. Thiagarajan, R. R., Barbaro, R. P., Rycus, P. T., Mcmullan, D. M., Conrad, S. A., Fortenberry, J. D., Paden, M. L., \& Centers, on behalf of the E. member. (2017). Extracorporeal Life Support Organization Registry International Report 2016. ASAIO Journal, 63(1),

60.https://doi.org/10.1097/MAT.0000000000000475

7. Greenberg, K., \& Kohl, B. (2018). ECMO used successfully in a near fatal case of opioid-induced acute respiratory distress syndrome. The American Journal of Emergency Medicine, 36(2), 343.e5-343.e6. https://doi.org/10.1016/j.ajem.2017.11.020

8. Wilson, M. W., Bonnecaze, A. K., Dharod, A., \& Miller, P.J. (2017). Analysis of Intensive Care Unit Admission and Sequelae in Patients Intravenously
Abusing Extended-Release Oral Oxymorphone. Southern Medical Journal, 110(3),

217-222.https://doi.org/10.14423/SMJ.0000000000000624

9. Hunt, R., Yalamanoglu, A., Tumlin, J., Schiller, T., Baek, J. H., Wu, A., Fogo, A. B., Yang, H., Wong, E., Miller, P., Buehler, P. W., \& Kimchi-Sarfaty, C. (2017). A mechanistic investigation of thrombotic microangiopathy associated with IV abuse of Opana ER. Blood, 129(7), 896-905. https://doi.org/10.1182/blood-2016-08-736579

10. Suresh, E. (2006). Diagnostic approach to patients with suspected vasculitis. Postgraduate Medical Journal, 82(970),

483-488.https://doi.org/10.1136/pgmj.2005.042648

11. Turesson, C., Schaid, D. J., Weyand, C. M., Jacobsson, L. T., Goronzy, J. J., Petersson, I. F., Dechant, S. A., Nyähll-Wåhlin, B.-M., Truedsson, L., Sturfelt, G., \& Matteson, E. L. (2006). Association of HLA-C3 and smoking with vasculitis in patients with rheumatoid arthritis. Arthritis and Rheumatism, 54(9), 2776-2783.https://doi.org/10.1002/art.22057

12. Ahmed, S. H., Aziz, T., Cochran, J., \& Highland, K. (2004). Use of extracorporeal membrane oxygenation in a patient with diffuse alveolar hemorrhage. Chest, 126(1),

305-309. https://doi.org/10.1378/chest.126.1.305

13. Suzuki, M., Yokota, T., Gomei, S., Saiki, T., Kamisasanuki, T., Hayamizu, A., Sugiki, D., \& Ikegami, K. (2016). Life-saving veno venous extracorporeal membrane oxygenation (VV-ECMO) in a case of potentially fatal massive hemoptysis due to bronchiectasis: A case report. Journal of the Japanese Society of Intensive Care Medicine, 23(3), 324-327.https://doi.org/10.3918/jsicm.23.324

14. Watts, R. A., Lane, S. E., Bentham, G., \& Scott, D. G. (2000). Epidemiology of systemic vasculitis: A ten-year study in the United Kingdom. Arthritis and Rheumatism, 43(2), 414-419. https://doi.org/10.1002/15290131(200002)43:2<414::AID-ANR23>3.0.CO;2-0

\section{Conflict of Interest: Nil}

Source of Support: None

\section{How to Cite this Article}

McGuire JA, McCarthy P, Herron R, Hayanga JWA, Nandwani V, Fugett J, Hayanga HK | Extracorporeal Life Support for Massive Hemoptysis and Acute Lung Injury Due to ANCA-Negative Vasculitis | Journal of Anaesthesia and Critical Care Case Reports | January-April 2021; 7(1): 04-07. 